\title{
Type 2 diabetes and dog walking: patients' longitudinal perspectives about implementing and sustaining physical activity
}

\author{
Elizabeth Peel, Margaret Douglas, Odette Parry and Julia Lawton
}

\begin{abstract}
Background

Physical activity is particularly important for people with type 2 diabetes, as evidence suggests that any reduction in sedentary time is good for metabolic health.

Aim

To explore type 2 diabetes patients' talk about implementing and sustaining physical activity.

Design of study

Longitudinal, qualitative study using repeat in-depth interviews with 20 patients over 4 years following clinical diagnosis.

Setting

Patients were recruited from 16 general practices and three hospitals across Lothian, Scotland.

Results

Discussion, and salience, of physical activity was marginal in patient accounts of their diabetes management. Patients claimed to have only received vague and non-specific guidance about physical activity from health professionals, and emphasised a perceived lack of interest and encouragement. Aside from walking, physical activities which were adopted tended to attenuate over time. Patients' accounts revealed how walking a dog assisted this kind of activity maintenance over time. Three main themes are highlighted in the analysis: 1) incidental walking; 2) incremental physical activity gains; and 3 ) augmenting physical activity maintenance. The problems arising from walking without a dog (for example, lack of motivation) are also examined.

\section{Conclusion}

Asking patients about pet preferences might seem tangential to medical interactions. However, encouraging dog walking or identifying another interest that promotes a regular commitment to undertake physical activity may yield long-term health benefits. Keywords

behaviour change; exercise; patients' perspectives; physical activity; qualitative study; type 2 diabetes.
\end{abstract}

\section{INTRODUCTION}

Physical activity has been described as the "best buy in public health'. ${ }^{1}$ Physical activity reduces the risk of a large number of health conditions and contributes to positive mental health. Yet fewer than $40 \%$ of adults achieve the recommended minimum level of activity, which is 30 minutes of moderate physical activity at least 5 days per week, ${ }^{2}$ or 60 minutes moderate activity most days for people who are trying to lose weight. Promotion of these levels of physical activity is particularly important for people with type 2 diabetes. Evidence suggests that any reduction in sedentary time is good for metabolic health, ${ }^{3}$ and that improvement in metabolic function (as measured by $\mathrm{HbA}_{1 \mathrm{c}}$ ) occurs when people with type 2 diabetes increase their levels of moderate activity. ${ }^{4}$

However, people with type 2 diabetes find it more difficult to engage in regular physical activity than they do modifying their diet, ${ }^{5}$ and most do not regularly undertake activity. ${ }^{6}$ It is only recently that interventions designed to ameliorate impaired glucose tolerance (IGT) or improve management of type 2 diabetes have seriously attempted to incorporate physical activity regimens alongside dietary advice or education.,8 Many organisations stress that exercise is 'a cornerstone of diabetes management'-11 and some authors recommend 'exercise prescription', ${ }^{12,13}$ although, evidence suggests that exercise referral

E Peel, PhD, senior lecturer in psychology, School of Life \& Health Sciences, Aston University, Birmingham. M Douglas, MSc, MRCGP, MFPHM, consultant in public health medicine, Lothian NHS Board, Edinburgh. O Parry, MSc, PhD, professor, Social Inclusion Research Unit, Glyndwr University, Plas Coch, Wrexham. J Lawton, PhD, senior research fellow, Population Health Sciences, University of Edinburgh, Edinburgh.

Address for correspondence

Elizabeth Peel, School of Life \& Health Sciences, Aston Triangle, Birmingham, B4 7ET. E-mail: e.a.peel@aston.ac.uk

Submitted: 20 November 2009; Editor's response: 28 January 2009; final acceptance: 23 March 2010.

(o) British Journal of General Practice 2010; 60: 570-577.

DOI: 10.3399/bjgp10X515061 
programmes are of limited effectiveness in promoting long-term changes in physical activity. ${ }^{10}$ Overall, evidence is lacking about how physical activity is implemented and sustained in the everyday lives of people with type 2 diabetes.

Recent qualitative studies embedded within randomised control trials (RCTs) have highlighted that, not only does increasing physical activity 'act as a gateway behaviour, that is, a behaviour that produces positive effects in other behaviours' such as diet,' but that 'increasing activity require(s) continual proactive effort'. ${ }^{8}$ Nonetheless, the longitudinal follow-up of participants in these studies was either limited to 9 months, ${ }^{7}$ or retrospective. ${ }^{8}$ While qualitative studies 'nested within' RCTs ${ }^{14}$ are clearly valuable, the experiences (and potentially motivation) of participants recruited to RCTs may differ from those undergoing routine health care. Furthermore, the types of intensive physical activity interventions implemented in RCTs are 'unlikely to be cost-effective within resource constrained healthcare systems'. ${ }^{14}$

Promoting physical activity, particularly among older adults, has been described as a 'formidable topic'. ${ }^{15}$ There is now extensive literature exploring perceived barriers or 'burdens' ${ }^{\prime}$ to increasing physical activity, both in older groups generally, ${ }^{15-17}$ and in people with type 2 diabetes in particular. ${ }^{6,18,19}$ Barriers that have been identified include: poor health, lack of time, ageing, adverse environments; ${ }^{17,20,21}$ safety/physical vulnerability, availability of facilities, cost, lack of motivation; ${ }^{16,20}$ cultural norms and social expectations; ${ }^{19}$ and difficulties exercising, tiredness, and being distracted by something good on television. ${ }^{18}$ While previous research has focused on perceived benefits as well as barriers to being physically active,,$^{16,22}$ the emphasis, to date, has tended to be on the latter. But it is unlikely that simply removing barriers is in itself sufficient to enable people to undertake physical activity. This study takes a different approach and examines the ways in which people living with type 2 diabetes talk about implementing and sustaining increased physical activity, through a repeat interview design. In doing so, a key aim was to explicate successes in diabetes self-management, and signal potential ways that physical activity could be incorporated and maintained in peoples' everyday lives.

\section{METHOD}

\section{Recruitment and participants}

Full details about recruitment are provided elsewhere..$^{23}$ In 200240 patients were recruited from the Lothian region of Scotland within 6 months of receiving a clinical diagnosis of type 2 diabetes. The sample was purposively selected to reflect the demographics of people with type 2 diabetes in

\section{How this fits in}

Increasing peoples' levels of physical activity is an important aspect of public

health policy, and regular physical activity promotes effective self-management

of chronic illnesses such as type 2 diabetes. There is limited understanding

about how physical activity is implemented and sustained in the everyday lives

of patients with type 2 diabetes. Patients' engagement in physical activities

other than walking decreases over time, and walking with a dog can result in

physical activity being sustained because dogs provide stimulus for regular

walking and companionship. Health professionals could discuss the benefits of

dog walking with patients, or work with patients to identify other interests that

could fulfil a similar function to that of dog walking.

Lothian, including patients receiving care in both primary and secondary settings. Participants were interviewed three times over 1 year (baseline, 6, 12 months) to explore their perceptions, experiences and adjustments made to their condition during the post-diagnostic period, ${ }^{24}$ and their education and support needs. ${ }^{25,26}$ Ethical approval was gained to contact participants after their third interviews, asking for permission to retain their contact details. Of the 37 participants who had not been lost to follow-up at the end of the first year, 21 gave consent for re-contact (Table 1). Twenty of these were interviewed a fourth time, 4 years later in 2006 (one had died in the interim). This fourth year anniversary was chosen to allow sufficient time to have elapsed for participants to have experienced changes in their diabetes and therefore, potentially, their approaches to disease self-management. ${ }^{27-30}$ There were no obvious differences between this sub-group and the original cohort in terms of demographic characteristics; a transition from predominately hospital-based to GP and nurse-led care occurred over the 4-year period. ${ }^{30}$

\section{Data collection}

To ensure continuity, all interviews were conducted by the first author. General topics included: participants' understandings of their diabetes and health; current treatments and views about regimen changes; and contact with services and professionals. Regarding physical activity, participants were asked:

- What, for you, counts as physical activity?

- How do you feel about physical activity compared to when I last saw you?

- What, if anything, are you doing to keep yourself physically active?

- What would you say hinders you from being physically active?

- What would you say encourages you to be physically active?

- Have any doctors or nurses encouraged you to be more physically active? 
Table 1. Participant information $(n=20)$.

\begin{tabular}{lc} 
Category & $n$ \\
\hline Age, years & \\
$40-49$ & 5 \\
$50-59$ & 1 \\
$60-69$ & 11 \\
$\geq 70$ & 3 \\
\hline Sex & \\
Female & 9 \\
Male & 11 \\
\hline
\end{tabular}

\begin{tabular}{ll}
\hline Socioeconomic status & \\
I-II & 6 \\
III N & 5 \\
III M & 8 \\
IV-V & 1 \\
\hline Treatment &
\end{tabular}

\begin{tabular}{lc}
\hline Round 1 & \\
Diet only & 9 \\
Tablets (monotherapy) & 11 \\
Tablets (combination therapy) & - \\
Insulin and Tablets & - \\
Round 4 & \\
Diet only & 6 \\
Tablets (monotherapy) & $5^{\circ}$ \\
Tablets (combination therapy) & 8 \\
Insulin and Tablets & 1 \\
\hline Location of Review & \\
\hline Round 1 & \\
Hospital & 16 \\
GP & 4 \\
Round 4 & \\
Hospital & 3 \\
GP & 15 \\
Shared care & 2 \\
\hline
\end{tabular}

${ }^{a}$ Mean age at last interview 60.8 years, range $40-80$ years. ${ }^{b}$ Using Registrar General's classification system. ${ }^{\circ}$ Three participants reported a dosage increase.

Interviews of 1-3 hours duration were conducted in participants' homes, tape-recorded with consent and transcribed verbatim. Field notes were also written immediately after each interview to capture initial impressions and additional contextual information. The longitudinal approach to data collection allowed for fruitful comparison between earlier and later rounds of interviews, and enabled participants' accounts of physical activity initiation, maintenance, or discontinuation to be analysed over time.

\section{Analysis}

This analysis adopted a critical realist epistemological position, treating participants' accounts as indicative of their lived 'reality' while recognising that the meanings attached to experiences are mediated by sociocultural contexts. ${ }^{31}$ The 80 interviews were repeatedly read and any talk pertaining to physical activity was coded using the qualitative software NVivo. Once these data were coded the field notes of the 20 participants were checked for relevant contextual information (for example, a participant wearing a t-shirt with a 'couch potato' motif, or an exercise bike being used as a clothes rack). Data were then re-read and data from participants who had implemented and sustained increases in physical activity were cross-compared with those who had not. Data from across participants' four interviews were then examined sequentially in order to explicate how, and why, approaches to physical activity changed over time. ${ }^{28,30}$ Data extracts are tagged with pseudonym, interview round (for example, 14), and other identifying information (for example, place names) has been changed.

\section{RESULTS}

In general, physical activity was not a key issue and the discussion, and salience, of physical activity was marginal in participants' accounts. Contrary to exercise being advocated as a 'cornerstone of diabetes management', few participants acknowledged that physical activity overall was fundamental to their diabetes self-care, and many communicated that they were:

'Not winning medals for the exercise stakes.'

(Eric, I2)

Participants claimed to have only received vague and non-specific guidance about physical activity from health professionals (if physical activity had been mentioned at all), and emphasised a perceived lack of interest and encouragement over time. ${ }^{32}$ For example:

'They don't ask you anything. They just say "are you getting exercise?" I says "Oh yes I get exercise". They don't enquire as to what type of exercise.' (Lindsay, 14)

'Well they'll ask, y'know, what exercise you get ... but they haven't said "Oh I think you should be walking twice as far" no, nothing like that.' (Duncan, 14)

Embedded in most participants' accounts was an awareness of the contemporary discourse stressing the health benefits of regular physical activity:

'Everybody knows that. Healthy lifestyle eh.' (Mary, 14)

'When you read the papers they say exercise is good for you.' (Walter, 14)

There was, however, limited appreciation articulated of the benefits of physical activity for blood glucose control: 
'They say light exercise is good for you, but I don't know if it helps with the diabetes or not.' (Walter, 14)

Only two participants intimated 'that [exercise] would burn off some of the sugar,' (Eric, I2) and only one suggested that this connection was communicated by health professionals:

'There was one doctor who once said to me back in the days when I was still on tablets that eh walking was a good way to get the blood sugar levels down.' (Callum, 14).

Walking, however, was the one activity that some participants described successfully adopting and sustaining over the 4 years:

'I will walk - a much more sedate approach to keeping fit.' (Callum, 14)

'I don't mind a bit [of a] walk, but that's the strength of my exercise.' (Walter, 14)

General views about physical activity and problematic aspects of physical activity maintenance are discussed before exploring the main themes. For those few participants who had successfully implemented and, importantly, maintained increases in their physical activity levels walking dogs played a vital role. The three ways that walking and dogs were enmeshed in participants' accounts were: 1) incidental walking; 2) incremental physical activity gains; and 3) augmenting physical activity maintenance.

\section{Difficulties implementing and maintaining physical activity}

Some participants characterised themselves as 'a couch potato' (Sadie, I2) or remarked that 'exercise equals pain' (Callum, 11). For most, any physical activity was incidental to their daily lives and consisted of domestic activities (for example, hoovering or gardening) or walking to achieve specific (non-health related) goals. As Allan said:

'I walk out and in to the pigeon loft at the back door, over to the shop for my cigarettes, paper.' Allan (14)

For those participants without responsibility for, or access to, a dog, their walking intentions were often not realised. For some, their 'opportunities' for walking were presented as limited:

I'm not a joiner [laughs] eh but I do go out with the walking group ... but it's only- well we do it once or twice a month.' (David, 14)

Or opportunities for walking were produced as reliant on external factors outside of their control. For example, Jennifer (aged 68) talked about regularly walking in her third interview with her neighbour, but by the fourth interview the neighbour had moved away and so it was less easy for them to walk together:

'I've got a new neighbour moved in a couple of doors down and she's into walking. So I'm walking quite- quite a- too much for me really y'know, but she says "you'll get used to it".' (Jennifer, I3)

'I had a neighbour two doors down and we used to walk a lot. We used to walk right round the country park and it took two hours on a Sunday morning, but she moved and it's not quite so easy now.' (Jennifer, 14)

For others without the routinising impetus of a dog, walking could be an unrealised good intention:

'I may be sitting here thinking here "I mean it's a nice day l'll go for a walk this afternoon".' (Sadie, I3).

Or the desire to deviate from the activity of walking would prove too tempting:

'I don't walk with Bob [husband] like we were going walking ... On a nice- when I say I walk up to [place] for the bus, if it's just me going up the town I'll walk to the next bus stop ... and then I have to walk up past [supermarket] "I'll just nip in and I'll get some milk. Oh, while I'm here I'll just have a coffee or maybe I'll just have my lunch instead" y'know [laughs]. So having been very good sweating the pounds off me l'm having an early lunch in [supermarket] and then I walk up to the bus stop [laughs].' (Sadie, 14)

For participants who had initially engaged in other forms of activity after being diagnosed with diabetes - such as exercise at a gym - 'it [had] faded out' (Frazer, 14) by the fourth interview. For others 'the novelty wore off' (Fiona, I2) much sooner, as Pauline eloquently described:

'I've got an exercise bike. It's the best ornament I ever bought.' (Pauline I2)

For some, increasing physical activity had only ever been a good intention: 
'My intention was to ... maybe go on a treadmill or something' (Walter, 14)

'I was going to buy a bike, but I don't really have anywhere to keep it' (Callum, 14).

\section{Incidental walking}

Having a dog, or having access to a dog, provided the extrinsic incentive for self-professed 'naturally lazy slob[s]' (Mary, I2) or participants who lacked intrinsic motivation to exercise to achieve and maintain regular physical activity.

Iris and Duncan, for example, both had their own dogs - dogs that pre-dated their diagnoses of type 2 diabetes. Iris (aged 49) classified herself as 'not really a very energetic person' (I1) and talked very little about physical activity throughout the interviews:

I'm not a gym person and I don't like swimming ... I mean going for a walk's fine, I can cope with that ... I went through spates of it when you go with all your pals to the gym and we all do this and that and then it falls through cos you all go to the pub afterwards and just [laughs] - that's the good bit [laughs].' (Iris, 14)

However, Iris had two dogs that she walked:

Interviewer (I): 'What, if anything, are you doing to keep yourself physically active?'

Iris 14: 'Apart from walking the dogs not a lot [laughs].'

Duncan (aged 66) differed from Iris in that he did not position himself as inherently opposed to physical activity. Although at the time of the fourth interview he was recuperating from an operation so was not engaging in his usual activities (for example, bowling or gardening). Nevertheless, the incidental physical activity generated by walking the dog was an enduring theme over the 4 years in Duncan's interviews:

'We've got a Labrador you've got to exercise every day. I have a good walk every day.' (I1)

'Oh yes, I mean that's exercised every day, every morning and the wife usually comes as well so we both get our exercise walking the dog.' (I2)

'I get plenty of exercise every day, you can see one of the reasons through there [points to the dog].' (I3)

In the fourth interview, despite his convalescence, the centrality of incidental walking was re-emphasised:

'We get regular exercise every day ... Rain, hail or shine ... We're out in all weathers aren't we [speaks to the dog].' (I4)

\section{Incremental physical activity gains}

Diane (aged 64) is a prototypical example of someone who struggled, because of multiple chronic illnesses (arthritis and anxiety), to undertake physical activity. She had difficulty merely 'doing my housework' (I1), and unfortunately she was unable to swim ('I'm scared of the water' [I2]):

'Because of the arthritis I don't exercise an awful lot ... like if I go for a walk er I have to watch how far I walk because I might be alright getting there, but l've got to remember that l've still got to get back.' (11)

'I don't do much exercise because, well mainly because I'm sore with the arthritis.' (I2)

'I'd like to be able to do more exercise, but I have arthritis so it's a problem. I'm limited to how much I can do but having said that ... I've been trying to go for a walk around the block.' (I3)

'I just go round the block. Occasionally eh like we do go to the park. Like Ralph [husband] only works in the mornings so in the afternoon I've seen us taking him [the dog] to the park and letting him have a run about with a ball.' (14)

I: 'I suppose your exercise - cos I remember when I spoke to you last about, well it would have been 3 years ago, and you were saying that you weren't - you wanted to do more than you could do?'

\section{Diane 14: 'Aha.'}

I: 'I mean mostly the arthritis I think is ... ?'

Diane 14: 'Aha, it's still the same.'

I: 'But then having said that if you've got him [dog] you are doing more than you were?'

Diane 14: 'Aha. Oh well I have to erm it makes me go out y'know.'

It was evident that while the health context for Diane had remained static over the intervening 3 years, a dog now provided a mandate for increased, and sustained, amounts of walking.

Mary (aged 52) had a chequered relationship with physical activity across the interviews, oscillating from emphatic rejection of formal exercise to attempting and failing to maintain increases in physical activity: 
'Every week I say "right, okay, this is the week you are going to do something" ... it hasn't happened ... l've no excuse. l've got a swimming pool at the bottom of my street ... The motivation's no[t] there! [laughs] ... I would never go to the likes of your aerobic classes or anything. I'm just not interested in things like that, Or going to the gym ... I call it Living Hell. Y'know, the "Living Well" gym! I said "Well it is to me!" ... It's just bloody living hell, who wants to go there y'know.' (11)

At the time of the second interview, 6 months later, Mary had joined a gym:

'... and when I come back from the gym I mean my count's down [blood glucose level] about 6 which apparently is normal n' that's obviously to do with exercise. So I know the answer. I just don't do it eh'. (I2)

However, her gym attendance had dwindled rapidly and she 'hated' much of the equipment:

'Och l've got to force myself. I'm not- well obviously l'm not a person who enjoys exercise let's be honest [laughs] erm or I wouldn't have ended up like this would I? I just hate it n' yon step thing, I hate that as well ... I did have this wild theory I would go three times a week. That lasted a week'. (12)

At the time of the third interview Mary had tried, and discontinued, swimming in the intervening 6 months, and her visits to the gym were increasingly sporadic:

'I'm still going, but maybe only- like l'll go one week and I'm thinking "ooph" ... It's not something I enjoy doing, it's as simple as that. It's like a chore.' (I3)

By the fourth interview, Mary had incorporated walking into her life:

I'm taking - not enough probably - but I'm certainly walking a heck of a lot more than I used [to]. Oh aye, I go out with my neighbour with her dog.' (14)

Although Mary's relationship with physical activity had not fundamentally changed - 'I'm not a very physically active person. I'm really not. Just I'm not into exercise. I've tried going to a gym and I hate it' her construction of walking was markedly different:

I like the walking, but not up mountains or anything. I don't mind going for a walk ... It's not a fast walk if you like, y'know we tend to stroll, but I reckon it's better than nothing ... I probably could do with going myself out walking quicker. But it's the motivation I lack.' (14)

Therefore, by the fourth interview, walking with her neighbour and her dog was crucial to the physical activity gains Mary had made since diagnosis. And, as she said herself, 'I reckon it's better than nothing' (14).

\section{Augmenting physical activity maintenance}

While most participants undertook less physical activity than recommended for manifold reasons, including lack of interest/motivation, unfacilitative shift work patterns, other chronic conditions (for example, angina or arthritis) one participant, Andy (aged 44), engaged in exemplary self-management behaviours consistently over the 4 years. ${ }^{33}$ Walking was Andy's primary form of physical activity, which was fully integrated into his daily routine before a year post-diagnosis:

'We go out for walks. I walk about 8 miles every single day ... 2 at lunchtime and 6 at night erm they'll [his children] often come with me at night ... It's not just a passing phase y'know that's the way it is now for as long as I'm able to draw breath basically erm because I want to keep on feeling this good ... it's just part of my life now erm and I would miss it if I stopped.' (I1)

'Still walking 6, 7, 8 miles a day erm every day ... It's just become a part of the routine that I enjoy. It's not a chore, it's something that I actually enjoy doing.' (I2)

'It all boils down to the fact that it was a novelty at first y'know and probably I honestly thought that "Och it won't last this exercise lark" y'know ... But it's got to the stage where I enjoy it and I almost need it at the end of the day ... just to get out for an hour or so, just on your own for reflection away from the noise of the children and people nagging [laughs]. (I3)

Andy 14: 'But the walking is catered for with the dog.'

I: 'Yes absolutely. And was that one of the reasons why you got her?'

Andy 14: 'No it was Heather [daughter] wanted a dog. So she did all the research and found the dog. So we agreed to take her knowing that the walking would very quickly pass down to me. But I walk every night anyway so erm it's good to 
have some canine company ... So I do between 7 and 8 miles every day, 7 days a week.'

Although initially Andy's children 'often' (11) accompanied him on his nightly walks, by the third interview quiet 'reflection' (I3) typified his habituated exercise routine. In the 3-year period between the third and fourth interview the family had got a dog. Therefore, in Andy's case, the 'good ... canine company' (14) augmented a pre-existing pattern of walking behaviour that he had successfully implemented and sustained prior to dog ownership.

\section{DISCUSSION}

\section{Summary of main findings}

Most participants in this study found regular physical activity challenging to maintain and 'faddy' forms of physical activity were particularly unsustainable. This analysis highlights that walking, ${ }^{21,34,35}$ - especially with a dog - is an achievable, and crucially, sustainable form of exercise for people with type 2 diabetes because it offers regular, routine activity and companionship. Other forms of physical activity had not been maintained by the fourth year after diagnosis.

\section{Strengths and limitations of the study}

A strength of this study is that it provides an in-depth, patient-centred, longitudinal perspective about physical activity maintenance. However, there is need for further research to understand the non-specific steers and the perceived lack of encouragement from health professionals identified in this study. Possible reasons might be health professionals feeling it is 'futile' to promote physical activity to patients or that advice about physical activity falls outside their remit; ${ }^{32}$ time constraints on doctor-patient interactions; or the division of labour within diabetes teams and the lack of a physical activity equivalent of a dietician. 'Lifestyle managed' rather than 'dietcontrolled' maybe a better descriptor for those recently diagnosed and/or those not using antiglycaemic agents or insulin.

\section{Comparison with existing literature}

Previous research has suggested that structured education programmes that incorporate pedometer use are effective for improving glucose tolerance in those with $\mathrm{IGT}^{36}$ and that self-efficacy and social support are linked to physical activity when community-based providers organise walking groups, ${ }^{37}$ Moreover, a systematic review of 48 intervention studies to promote walking (including brief advice, remote support [telephone/internet], group- and community-based approaches and pedometer use) suggested that 'we do not yet know whether or how the benefits of individual and group level [walking] interventions shown to be effective in ... the short term can be sustained' (the current authors' emphasis). ${ }^{34}$ This longitudinal study goes some way to addressing this issue and emphasises the efficacy of dog walking as a daily practice.

\section{Implications for clinical practice}

This study's findings indicate some avenues for health professionals to explore in routine practice regarding physical activity. Ascertaining patients' preferences, and any constraints (psychological, physical and geographical), on physical activity could be a useful segue into tailoring advice and appropriate goal setting. Health professionals should more regularly and explicitly raise physical activity as one element in improving diabetes control, and the mitigating influence of physical activity on secondary complications could be emphasised.

Asking patients about pet preferences may seem tangential to medical interactions. Yet, these findings highlight the important role that dogs can play in sustaining physical activity over time. While recommending dog walking may not be appropriate for all patients (indeed, in some instances, it may be culturally and/or religiously insensitive), given the average lifespan of a dog is over 10 years the potential for physical activity maintenance is great. Exploring with patients whether it would be practicable, or indeed desirable, for them to own a dog (or offer to walk a dog for a friend or from a pet rescue charity) could have long-term physical health benefits.

\section{Funding}

This study was funded by the Chief Scientist Office, Scottish Executive Health Department (CZH/4/41 and CZG/2/241). The researchers' work was independent of the funding body. Ethical approval

NHS Lothian Research Ethics Committees approval was granted for the study (LREC//2001/3/20).

\section{Competing interests}

The authors have stated that there are none.

\section{Acknowledgements}

With thanks to the people with diabetes who took part in the study, the health professionals who assisted with recruitment, and Margaret MacPhee for her administrative and secretarial support.

\section{Discuss this article}

Contribute and read comments about this article on the Discussion Forum: http://www.rcgp.org.uk/bjgp-discuss

\section{REFERENCES}

1. Morris JN. Exercise in the prevention of coronary heart disease: today's best buy in public health. Med Sci Sport Exerc 1994; 26(7): 807-814.

2. Physical Activity Task Force. Let's make Scotland more active: a strategy for physical activity. Edinburgh: Crown Copyright, 2003. http://www.scotland.gov.uk/library5/culture/lmsa-00.asp (accessed 21 Jun 2010)

3. Helmerhorst HJF, Wijndaele K, Brage S, et al. Objectively measured sedentary time may predict insulin resistance independent of moderate- and vigorous-intensity physical activity. Diabetes 2009; 
58(8): 1776-1779.

4. Hordern MD, Coombes JS, Cooney LM, et al. Effects of exercise intervention on myocardial function in type 2 diabetes. Heart 2009 95(16): 1343-1349.

5. Mosnier-Pudar H, Hochberg G, Eschwege E, et al. How do patients with type 2 diabetes perceive their disease? Insights from the French DIABASIS survey. Diabetes Metab 2009; 35(3): 220-227.

6. Hays LM, Clark DO. Correlates of physical activity in a sample of older adults with type 2 diabetes. Diabetes Care 1999; 22(5): 706-712.

7. Malpass A, Andrews R, Turner KM. Patients with type 2 diabetes experiences of making multiple lifestyle changes: a qualitative study. Patient Educ Couns 2009; 74(2): 258-263.

8. Penn L, Moffatt SM, White M. Participants' perspective on maintaining behaviour change: a qualitative study within the European Diabetes Prevention Study. BMC Public Health 2008; 8: 235.

9. Sigal RJ, Kenny GP, Wasserman DH, et al. Physical activity/exercise and type 2 diabetes - a consensus statement from the American diabetes association. Diabetes Care 2006; 29(6): 1433-1438.

10. National Institute of Health and Clinical Excellence. Four commonly used methods to increase physical activity: brief interventions in primary care, exercise referral schemes, pedometers and community-based exercise programmes for walking and cycling. Public Health Intervention Guidance no 2. London: NICE, 2006.

11. Gavi S, Hensley J. Diagnosis and management of type 2 diabetes in adults: a review of the ICSI guideline. Geriatrics 2009; 64(6): 12-17.

12. Okay DM, Jackson PV, Marcinkinewicz M, Papino MN. Exercise and obesity. Prim Care 2009; 36(2): 379-393.

13. Pedersen BK, Saltin B. Evidence for prescribing exercise as therapy in chronic disease. Scand J Med Sci Spor 2006; 16 (Suppl 1): 3-63.

14. Daley AJ, Copeland RJ, Wright RJ, Wales JKH. 'I can actually exercise if I want to; it isn't as hard as I thought': a qualitative study of the experiences and views of obese adolescents participating in an exercise therapy intervention. J Health Psychol 2008; 13(6): 810-819.

15. Brawley LR, Rejeski WJ, King AC. Promoting physical activity for older adults: the challenges for changing behavior. Am J Prevent Med 2003; 25(3 Suppl 2): 172-183.

16. O'Brien Cousins S. 'My heart couldn't take it': older women's belief about exercise benefits and risks. J Gerontol B Psychol Sci Soc Sci 2000; 55(5): 283-294.

17. Grossman MD, Stewart AL. 'You aren't going to get better by just sitting around': physical activity perceptions, motivations, and barriers in adults 75 years of age or older. Am J Geriatric Cardiology 2003; 12(1):33-37.

18. Thomas N, Alder E, Leese GP. Barriers to physical activity in patients with diabetes. Postgrad Med J 2004; 80(943): 287-291.

19. Lawton J, Ahmad N, Hanna L, et al. 'I can't do any serious exercise': barriers to physical activity among people of Pakistani and Indian origin with type 2 diabetes. Health Educ Res 2006; 21(1): 43-54.

20. Eyler AA, Baker E, Cromer L, et al. Physical activity and minority women: a qualitative study. Health Educ Behav 1998; 25(5): 640-652.

21. Booth ML, Bauman A, Owen N, Gore CJ. Physical activity preferences, preferred sources of assistance, and perceived barriers to increased activity among physically inactive Australians. Prevent Med 1997; 26(1): 131-137.

22. Juarbe T, Turok XP, Pérez-Stable EJ. Perceived benefits and barriers to physical activity among older Latina women. Western J Nurs Res 2002; 24(8): 868-886.

23. Peel E, Parry O, Douglas M, Lawton J. 'It's no skin off my nose': why people take part in qualitative research. Qual Health Res 2006; 16(10): 1335-1349.

24. Lawton J, Parry O, Peel E, Douglas M. Diabetes service provision: a qualitative study of newly diagnosed type 2 diabetes patients' preferences and views. Diabet Med 2005; 22(9): 1246-1251.

25. Lawton J, Peel E, Parry O, et al. Lay perceptions of type 2 diabetes in Scotland: bringing health services back in. Soc Sci Med 2005; 60(7): 1423-1435.

26. Peel E, Parry O, Douglas M, Lawton J. Diagnosis of type 2 diabetes: a qualitative analysis of patients' emotional reactions and views about information provision. Patient Educ Couns 2004; 53(3): 269-275.

27. Peel E, Douglas M, Lawton J. Self-monitoring of blood glucose in typ 2 diabetes: longitudinal qualitative study of patients' perspectives. $B M$ J 2007; 335(7618): 493-498.

28. Lawton J, Peel E, Douglas M, Parry O. Shifting accountability: a longitudinal qualitative study of diabetes causation accounts. Soc Sci Med 2008; 67(1): 47-56.
29. Lawton J, Peel E, Parry O, Douglas M. Patients' perceptions and experiences of taking oral glucose lowering agents: a longitudinal qualitative study. Diabet Med 2008; 25(4): 491-495.

30. Lawton J, Rankin I, Peel E, Douglas M. Patients' perceptions and experiences of transitions in diabetes care: a longitudinal qualitative study. Health Expect 2009; 12(2): 138-148.

31. Willig C. Beyond appearances: a critical realist approach to social constructionism. In: DJ Nightingale, J Cromby (eds). Social constructionist psychology: a critical analysis of theory and practice. Buckingham: Open University Press, 1999: 37-51.

32. Epstein L, Ogden J. A qualitative study of GPs' views of treating obesity. Br J Gen Pract 2005; 55(519): 750-754.

33. Parry O, Peel E, Douglas M, Lawton J. Issues of cause and control in patient accounts of type 2 diabetes. Health Educ Res 2006; 21(1): 97-107.

34. Ogilvie D, Foster CE, Rothnie $\mathrm{H}$, et al. Interventions to promote walking: systematic review. BMJ 2007; 334(7605): 1204-1213.

35. Wandel M, Roos G. Age perceptions and physical activity among middle-aged men in three occupational groups. Soc Sci Med 2006 62(12): 3024-3034

36. Yates $\mathrm{T}$, Davies M, Gorely $\mathrm{T}$, et al. Effectiveness of a pragmatic education program designed to promote walking activity in individuals with impaired glucose tolerance: a randomized controlled trial. Diabetes Care 2009; 32(8): 1404-1410.

37. Ingram M, Ruiz M, Mayorga MT, Rosales C. The Animadora Project: identifying factors related to the promotion of physical activity among Mexican Americans with diabetes. Am J Health Promot 2009; 23(6): 396-402. 